\title{
Visualization of the Peripheral Branches of the Mandibular Division of the Trigeminal Nerve on 3D Double-Echo Steady-State with Water Excitation Sequence
}

\author{
H. Fujii, A. Fujita, A. Yang, H. Kanazawa, K. Buch, O. Sakai, and H. Sugimoto
}

\begin{abstract}
BACKGROUND AND PURPOSE: Although visualization of the extracranial branches of the cranial nerves has improved with advances in MR imaging, only limited studies have assessed the detection of extracranial branches of the mandibular nerve (V3). We investigated the detectability of the branches of $\mathrm{V} 3$ on a 3D double-echo steady-state with water excitation sequence.
\end{abstract}

MATERIALS AND METHODS: We retrospectively evaluated the detectability of the 6 branches of the V3, the masseteric, buccal, auriculotemporal, lingual, inferior alveolar, and mylohyoid nerves, by using a 5-point scale (4, excellent; 3, good; 2, fair; 1, poor; and 0, none) in 86 consecutive patients who underwent MR imaging with the 3D double-echo steady-state with water excitation sequence. Weighted $\kappa$ analysis was used to calculate interobserver variability among the 3 readers.

RESULTS: The detection of the lingual and inferior alveolar nerves was the most successful, with excellent average scores of 3.80 and 3.99 , respectively. The detection of the masseteric, the buccal, and the auriculotemporal nerves was good, with average scores of 3.31, 2.67, and 3.11 , respectively. The mylohyoid nerve was difficult to detect with poor average scores of 0.62 . All nerves had excellent interobserver variability across the 3 readers (average weighted $\kappa$ value, 0.95-1.00).

CONCLUSIONS: The 3D double-echo steady-state with water excitation sequence demonstrated excellent visualization of the extracranial branches of $\mathrm{V} 3$ in most patients. The 3D double-echo steady-state with water excitation sequence has the potential for diagnosing $\mathrm{V} 3$ pathologies and preoperatively identifying peripheral cranial nerves to prevent surgical complications.

ABBREVIATIONS: 3D-DESS-WE $=3$ D double-echo steady-state with water excitation; $\mathrm{V} 3=$ mandibular nerve (third division of the trigeminal nerve)

$\mathbf{C}_{\mathrm{p}}^{\mathrm{r}}$ anial nerve deficits are not uncommon, and there are many pathologic processes that can affect the cranial nerves. ${ }^{1-10}$ Unfortunately, the physical examination findings are often nonspecific for differentiating among these pathologic causes, and imaging plays a crucial role in diagnosing pathologic processes affecting the cranial nerves. With increasing spatial and contrast resolution of cross-sectional imaging, better visualization of the cranial nerves and their major branches has become possible, but the delineation of the entire course of the extracranial segments of the cranial nerves still remains a diagnostic challenge. ${ }^{11-17}$

Received October 30, 2014; accepted after revision December 17

From the Department of Radiology (H.F., A.F., H.K., H.S.), Jichi Medical University School of Medicine, Tochigi, Japan; Departments of Radiology (A.F., K.B., O.S.), Otolaryngology-Head and Neck Surgery (O.S.), and Radiation Oncology (O.S.), Boston Medical Center, and Boston University School of Medicine (A.Y.), Boston, Massachusetts.

Paper previously presented at: Annual Meeting of the American Society of Head and Neck Radiology, September 10-14, 2014; Seattle, Washington.

Please address correspondence to Akifumi Fujita, MD, Department of Radiology, Jichi Medical University School of Medicine, 3311-1, Yakushiji, Shimotsuke, Tochigi 329-0498, Japan; e-mail: akifuji@jichi.ac.jp

http://dx.doi.org/10.3174/ajnr.A4288
The trigeminal nerve has the largest distribution of innervation among all the cranial nerves in the suprahyoid neck. Even though the mandibular nerve (V3) is the largest division of the trigeminal nerve, there have been only limited studies investigating the visualization of the extracranial segments of V3 with MR imaging. Several prior studies have focused on imaging the extracranial segments of V3 by using a T1-weighted fast-spoiled gradient recalled-echo sequence with fat suppression, ${ }^{9}$ a T1weighted MPRAGE sequence with water excitation fat suppression, ${ }^{18}$ or a diffusion tensor tractography sequence, ${ }^{19}$ but these studies evaluated only the inferior alveolar nerve. Another study used FIESTA and fast-spoiled gradient recalled-echo sequences to evaluate the entire V3 nerve, but the extracranial peripheral V3 branches were not well-demonstrated, with the exception of the inferior alveolar and lingual nerves. ${ }^{13}$

The 3D double-echo steady-state with water excitation (3DDESS-WE) sequence is a recently introduced MR imaging technique that can delineate the peripheral cranial nerves as highsignal-intensity structures. ${ }^{20}$ At our institution, this sequence has been added to our standard MR imaging protocol of the salivary 

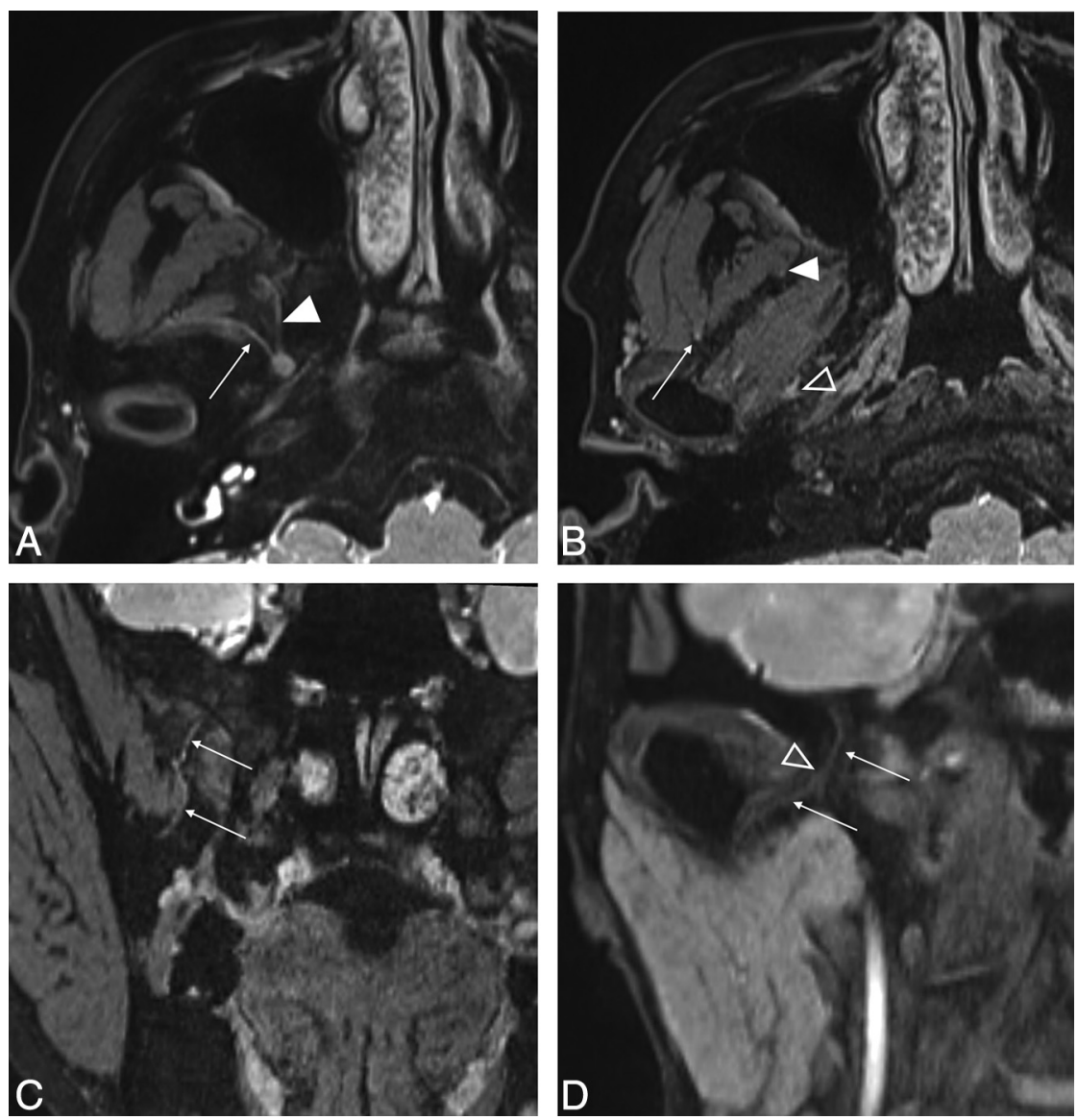

ation. Ultimately, 85 patients were included in this study, and there were certain patients whose respective nerve branches were excluded from the evaluation.

\section{MR Imaging Procedures}

All patients underwent MR imaging with a 3T unit (Magnetom Skyra; Siemens, Erlangen, Germany) by using a commercially available 20 -channel head-neck coil with our routine neck protocol that included the 3D-DESS-WE sequence. The precise parameters of the 3D-DESS-WE sequence are as follows: $\mathrm{TR} / \mathrm{TE}=11 / 4.21$ $\mathrm{ms}$, flip angle $=30^{\circ}, \mathrm{FOV}=200 \times 200$ $\mathrm{mm}$, matrix $=384 \times 244$, effective section thickness $=0.82 \mathrm{~mm}$, number of acquisitions $=1$, scan time $=4$ minutes $12 \mathrm{sec}$ onds. Slab thickness $(11.2 \mathrm{~cm})$ of the 3DDESS-WE sequence is from the skull base to the mandible.

\section{Image Evaluation}

All acquired images were transferred to our clinical server and evaluated with a PACS, with a 2-megapixel high-resolution liquid-crystal display. Three readers (reader A, third- year radiology resident; reader $\mathrm{B}$, first-year medical student; reader $\mathrm{C}$, attending neuroradiologist with 17 years of experience) independently evaluated the 6 extracranial branches of the V3: the masseteric nerve, the buccal nerve, the auriculotemporal nerve, the lingual nerve, the inferior alveolar nerve, and the mylohyoid nerve. The courses of the V3 branches were

glands and has been used routinely to evaluate the intraparotid facial nerve and salivary ducts within the salivary glands since October 2012. The purpose of this study was to investigate the detectability of the extracranial peripheral branches of V3 on the 3D-DESS-WE sequence.

\section{MATERIALS AND METHODS \\ Patient Population}

This retrospective study was approved by our institutional review board, and informed consent was waived. We reviewed 86 consecutive patients who underwent MR imaging for the evaluation of salivary gland lesions from October 2012 to March 2014 (40 males and 46 females; age range, $17-88$ years; mean age, 58 years; median age, 63 years). Of 86 patients, the final diagnoses or suspected diagnoses were as follows: 47 benign tumors, 6 cases of Sjögren syndrome, 5 infectious diseases, 5 malignant tumors, and 5 submandibular lesions; the rest of the 18 patients were healthy or had lesions incidentally found in other locations. Patients with severe motion artifacts, metal artifacts, or large mass lesions deforming adjacent anatomic structures were excluded from evalu- determined by tracing the nerve from the foramen ovale distally, compared with anatomic diagrams from Gray's Anatomy. ${ }^{21}$ Axial source images of the 3D-DESS-WE sequence were mainly used for tracing the branches of the V3. Reconstructed sagittal and coronal thin (3-mm thick) slab MIP images of the 3D-DESS-WE sequence were also used simultaneously.

Representative images of the 6 branches of V3 are shown in Figs 1 and 2. As the V3 descends from the foramen ovale, it divides into the anterior division, which gives off the masseteric nerve and the buccal nerve (Fig 1A), and the posterior division, which gives off the auriculotemporal nerve (Fig 1D), the lingual nerve (Fig $2 A$ ), the inferior alveolar nerve (Fig $2 B$ ), and the mylohyoid nerve (Fig 2C).

We evaluated the images focusing on the continuity of each V3 branch and distinguishing the branch from the $\mathrm{V} 3$ trunk to avoid potential confusion of vascular structures for nerve structures. Each of the 6 branches of $\mathrm{V} 3$ was divided into proximal and distal portions at intermediate points to facilitate evaluation. The intermediate point for the masseteric nerve is the point at which the 

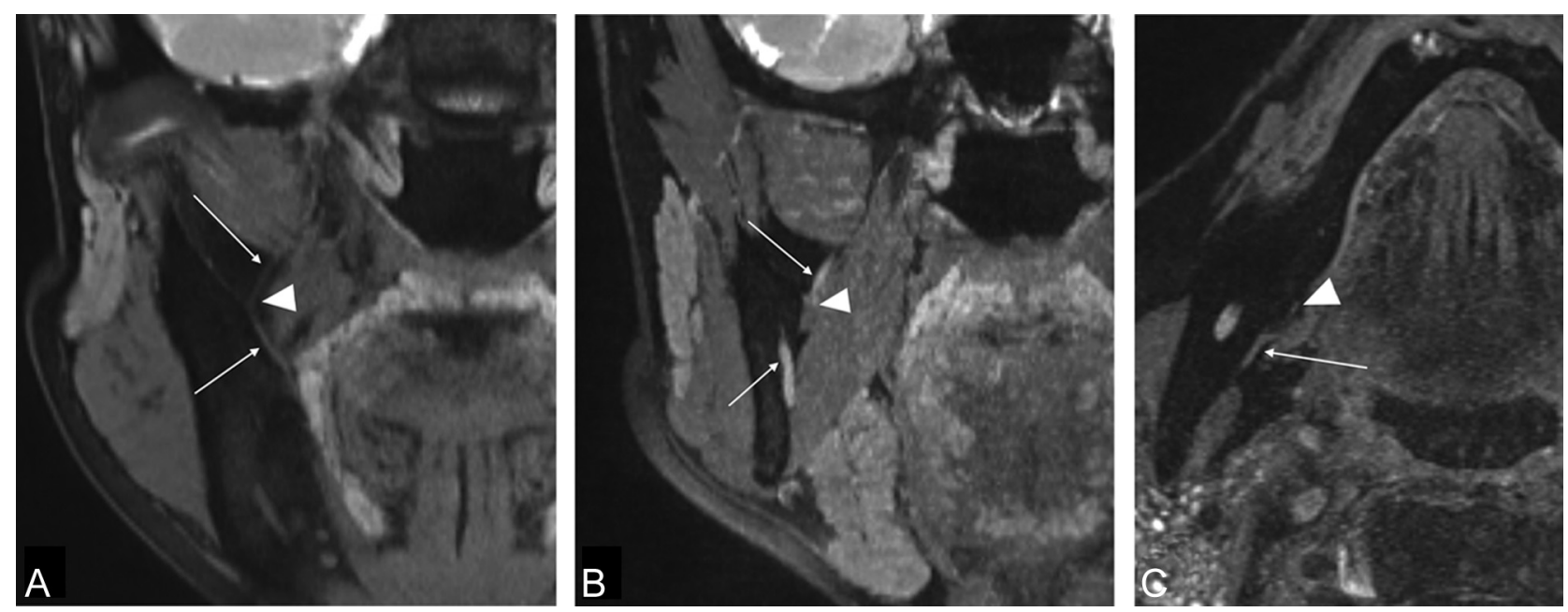

FIG 2. $A$ and $B$, Coronal reformatted image of the 3D-DESS-WE sequence. $C$, Axial source image of the 3D-DESS-WE sequence. Reformatted coronal images show the lingual nerve ( $A$, arrows) and the inferior alveolar nerve ( $B$, arrows). The intermediate points are established where the lingual nerve running laterally starts to change direction medially ( $A$, arrowhead) and where the inferior alveolar nerve enters the mandibular foramen ( $B$; arrowhead). Axial image shows that the mylohyoid nerve runs at the medial surface of the mandible ( $C$, arrow). The intermediate point is established where the nerve enters the mylohyoid muscle ( $C$, arrowhead).

nerve enters the deep surface of the masseter muscle (Fig $1 B$ ). The intermediate point for the buccal nerve is where the nerve enters the anterolateral edge of the lateral pterygoid muscle (Fig 1B). On the coronal image, the distal portion of the buccal nerve runs an $\mathrm{S}$-shaped course (Fig 1C). The intermediate point for the auriculotemporal nerve is where the nerve enters the pterygoid venous plexus (Fig $1 B,-D$ ). The intermediate point for the lingual nerve is where the nerve running laterally starts to change direction medially (Fig 2A). The intermediate point for inferior alveolar nerve is where the nerve enters the mandibular foramen (Fig $2 B$ ). The intermediate point for the mylohyoid nerve is where the nerve enters the mylohyoid muscle (Fig 2C).

\section{Statistical Analysis}

We evaluated the capability of detecting the V3 branches with a 5-point scale with the criteria as follows: excellent (4), both the proximal and distal portions of the nerve identified; good (3), both the proximal and distal portions of the nerve identified but not continuous; fair (2), only the proximal portion of the nerve identified; poor (1), only the proximal portion of the nerve identified but not continuous; and none (0), the nerve not identified. The average scores for each reader, in addition to the average score from all 3 readers for the 6 branches of the V3, were calculated.

The weighted $\kappa$ analysis used to calculate interobserver variability was defined as follows: poor, $\leq 0.2$; fair, $>0.2$ to $\leq 0.4$; moderate, $>0.4$ to $\leq 0.6$; good, $>0.6$ to $\leq 0.8$; and excellent, $>0.8$ to $\leq 1 .{ }^{22}$

\section{RESULTS}

The 3D-DESS-WE sequence was performed in all 86 patients, but 1 patient was excluded because of severe motion artifacts. With 85 patients, 170 nerves for each side of the $\mathrm{V} 3$ were reviewed. Because patients with metal artifacts and large mass lesions deforming the adjacent anatomic structures were excluded from the evaluation, 166 masseteric nerve, 166 buccal nerve, 167 auriculotemporal nerve, 159 lingual nerve, 159 inferior alveolar nerve, and 159 mylohyoid nerve segments were included in the analysis.
The average evaluation scores performed by the 3 readers and the interobserver variability are shown in Tables 1 and 2 .

The detection of the lingual and the inferior alveolar nerves was excellent, with average scores of 3.80 and 3.99, respectively. The detection of the masseteric, buccal, and auriculotemporal nerves was relatively good, with average scores of 3.31, 2.67, and 3.11 , respectively. The mylohyoid nerve was difficult to detect, with an average score of 0.62 .

The interobserver variability was excellent among the 3 readers (average weighted $\kappa$ value, $0.95-1.00$ ).

\section{DISCUSSION}

Because the V3 is a large cranial nerve with mixed sensory and motor function that innervates a large region of the suprahyoid neck, a wide variety of physical manifestations and pathologies such as tumor, inflammatory/infectious disease, vascular disease, and trauma can be found affecting it in clinical practice. ${ }^{7,11,12} \mathrm{~V} 3$ contains clinically important branches often affected by head and neck cancers via direct invasion and/or perineural tumor spread. All of these pathologic etiologies have the potential to impact clinical outcomes significantly and may contribute to significant morbidity and quality of life. ${ }^{3,10,23,24}$ An in-depth understanding of the anatomy and normal course of the extracranial nerves is required to make the diagnosis of many of these pathologies, and more sophisticated and dedicated high-resolution MR images for peripheral cranial nerve imaging are expected to enter and influence clinical practice.

The widespread uses of volumetric CT scanners and high-field MR imaging scanners and recent advances in MR imaging technology have provided opportunities to obtain high-resolution images of the cranial nerves. Following the entire course of the cranial nerves, including the extracranial segments, however, is still a diagnostic challenge in routine clinical practice. ${ }^{1,2,11-16}$ Intracranial segments of the cranial nerves, particularly the cisternal segments, are readily detected by using high-resolution heavily T2-weighted imaging. ${ }^{14,15}$ Without the high-signal background from the CSF, however, heavily 
Table 1: Average scores of the 3 readers evaluating the V3 nerves

\begin{tabular}{lcccc}
\hline \multicolumn{1}{c}{ V3 Nerves } & Reader A & Reader B & Reader C & Average \\
\hline Masseteric & $3.34 \pm 1.083$ & $3.36 \pm 1.015$ & $3.24 \pm 1.016$ & $3.31 \pm 1.038$ \\
Buccal & $2.64 \pm 1.308$ & $2.75 \pm 1.332$ & $2.63 \pm 1.281$ & $2.67 \pm 1.306$ \\
Auriculotemporal & $3.13 \pm 1.376$ & $3.07 \pm 1.220$ & $3.13 \pm 1.185$ & $3.11 \pm 1.261$ \\
Lingual & $3.77 \pm 0.781$ & $3.88 \pm 0.544$ & $3.75 \pm 0.682$ & $3.80 \pm 0.677$ \\
Inferior alveolar & $4.00 \pm 0.000$ & $4.00 \pm 0.000$ & $3.97 \pm 0.157$ & $3.99 \pm 0.091$ \\
Mylohyoid & $0.46 \pm 0.785$ & $0.67 \pm 0.917$ & $0.72 \pm 0.764$ & $0.62 \pm 0.831$ \\
\hline
\end{tabular}

Table 2: Interobserver variability of the 3 readers (A, B, and C) evaluating the V3 nerves

\begin{tabular}{lcccc}
\hline \multicolumn{1}{c}{ V3 Nerves } & A and B & B and C & C and A & Average \\
\hline Masseteric & 0.960 & 0.970 & 0.961 & 0.96 \\
Buccal & 0.954 & 0.973 & 0.936 & 0.95 \\
Auriculotemporal & 0.942 & 0.961 & 0.955 & 0.95 \\
Lingual & 0.974 & 0.994 & 0.977 & 0.98 \\
Inferior alveolar & 1.000 & 0.998 & 0.998 & 1.00 \\
Mylohyoid & 0.983 & 0.967 & 0.959 & 0.97 \\
\hline
\end{tabular}

T2-weighted imaging has not proved to be very useful in visualizing the extracranial segments of the cranial nerves. Detecting the remaining peripheral segments can be achieved with pre- and postcontrast high-resolution 3D T1-weighted images (gradient-recalled acquisition in steady state, fast-spoiled gradient recalled-echo, or MPRAGE) with and without fat suppression, ${ }^{9-13,16,18,23}$ but detecting the entire course of the extracranial branches of the cranial nerves is still very challenging.

The 3D-DESS-WE sequence, commonly used in musculoskeletal imaging, was recently applied for visualization of the intraparotid facial nerve and is quite successful. ${ }^{20}$ In our institution, we routinely use the 3D-DESS-WE sequence when investigating parotid lesions, both for visualization of the intraparotid facial nerve to localize the parotid tumor and to detect involvement of the nerve. In this study, we assessed the detectability of the extracranial segments of $\mathrm{V} 3$ by using the 3D-DESS-WE sequence.

Our study demonstrates that it is possible to detect the entire course of the main branches of V3 with non-contrast-enhanced MR imaging. In a recent study performed by Cassetta et $\mathrm{al},{ }^{13}$ the detectability of the branches of the V3 (buccal, auriculotemporal, lingual, and inferior alveolar nerves) was evaluated with a combination of FIESTA and fast-spoiled gradient recalled-echo sequences performed with a $3 \mathrm{~T}$ scanner, but the authors were not able to identify the buccal nerve or the auriculotemporal nerve in all cases. The 3D-DESS-WE sequence used in this study demonstrated superior detection of the 4 branches of the V3, including the buccal and auriculotemporal nerves. This study also builds on prior work in which we assessed the detectability of the masseteric and the mylohyoid nerves. ${ }^{13}$

Because the lingual and the inferior alveolar nerves are surrounded by fat and/or fatty bone marrow along their course, the detection of these nerves was excellent by using a water excitation fat-suppression technique. ${ }^{20}$ The detection of the masseteric and the buccal nerves was also relatively good. The 3D-DESS-WE sequence involves the acquisition of 2 different echoes during each TR, on the basis of the steady-state free precession technique. The first echo is the free induction decay gradient echo used in the FISP sequence, and the second is the spin-echo used in the steadystate free precession sequence. ${ }^{20}$ Because the steady-state free precession signal intensity has a dominant T2 contrast $^{17}$ and the FISP signal intensity provides more anatomic details with tissue contrast dominated by the $\mathrm{T} 1 / \mathrm{T} 2$ ratio, ${ }^{20}$ we speculate that the component of $\mathrm{T} 2$ contrast in the DESS sequence would show the nerve itself as a high-signal-intensity structure and would enable us to track the nerve easily with paging methods on the PACS viewer. In future studies, we expect that the T2 contrast of the nerve itself will enable the detection of signal abnormalities in nerves with normal morphology, such as the early stage of the perineural spread of malignancy and infection/inflammatory disease.

Although it was difficult to differentiate the auriculotemporal nerve from the pterygoid venous plexus in some patients, the detection of the auriculotemporal nerve was relatively successful in our study. Because the auriculotemporal nerve is often involved in the perineural spread of malignancy, ${ }^{3,10,24}$ our results suggest that the $3 \mathrm{D}$ DESS-WE sequence is a promising method for evaluating perineural tumor spread. The main explanation for the poor detection of small branches is that the visualization depends on the size of the nerve. To better visualize small nerves such as the mylohyoid nerve, further refinement in the sequence design or higher magnet strength for higher SNR may be needed. Size was not the sole limitation for the detection of small nerves, however, because the masseteric, buccal, and auriculotemporal nerves were visualized well in this study. The visibility of small nerve structures may depend on the morphology and arrangement of structures adjacent to each nerve branch. Relatively lower scores of masseteric and buccal nerves compared with the auriculotemporal nerve are likely secondary to susceptibility artifacts from the sinonasal cavity air.

In this study, we evaluated the capability of depicting the segments of the V3 branches by using 3 readers of dramatically different backgrounds and levels of training: a medical student, a radiology resident, and a neuroradiology attending physician. The interobserver variabilities among the 3 readers were excellent; this outcome reflected the inherent improved diagnostic capabilities of the V3 nerves by using the 3D-DESS-WE sequence. Although the reader's experience influences the imaging interpretation and impacts the diagnostic assessment, we believe that interpreting images by tracking the nerves in this $3 \mathrm{D}$-DESS-WE sequence is a promising method for detecting and evaluating the extracranial peripheral cranial nerves. The advantages of the 3D-DESS-WE sequence include its short acquisition time and using the standard head and neck coil without a surface coil, in addition to high and uniform detectability of the peripheral cranial nerves by readers at various training levels. These features make the 3D-DESS-WE sequence readily feasible in routine clinical practice.

One of the limitations of this study was the presence of susceptibility and motion artifacts, which were prominent in the $3 \mathrm{D}$ DESS-WE sequence, and inhomogeneous fat suppression, which interfered with visualization of the nerves. We noticed that the 
masseteric and buccal nerves are affected by the susceptibility effect from the paranasal sinus air. Applying other types of fatsuppression techniques to the $3 \mathrm{D}$ double-echo steady-state sequence may improve the visualization of the nerves. Another limitation is the subjective and qualitative evaluation used in this study; objective quantitative evaluation may be needed to discriminate normal and abnormal findings of the nerves in a future study. Finally, because we have no surgical or pathologic correlation with images in this study, we cannot tell whether the smallsized nerve branches are actually the small-sized nerves, but instead we relied on their having a characteristic anatomic course.

\section{CONCLUSIONS}

The 3D-DESS-WE sequence demonstrated excellent visualization of the extracranial branches of the V3, such as the masseteric nerve, buccal nerve, auriculotemporal nerve, lingual nerve, and inferior alveolar nerve, in most patients. Uniform high detectability of the normal course of peripheral branches of the V3 was obtained by readers with very different experience. The 3DDESS-WE sequence has the potential for diagnosing V3 pathologies and for more precise preoperative identification of the peripheral nerves, which may help in preventing complications from surgical intervention with future studies.

Disclosures: Osamu Sakai-UNRELATED: Payment for Lectures (including service on Speakers Bureaus): Bracco Diagnostics, Kyorin USA, Eisai, Comments: honoraria for lectures; Royalties: McGraw-Hill.

\section{REFERENCES}

1. Borges A, Casselman J. Imaging the cranial nerves. Part I. Methodology, infectious and inflammatory, traumatic and congenital lesions. Eur Radiol 2007;17:2112-25

2. Borges A, Casselman J. Imaging the cranial nerves. Part II. Primary and secondary neoplastic conditions and neurovascular conflicts. Eur Radiol 2007;17:2332-44

3. Chan $\mathrm{M}$, Dmytriw AA, Bartlett $\mathrm{E}$, et al. Imaging of auriculotemporal nerve perineural spread. Ecancermedicalscience 2013;7:374

4. Ginsberg LE, DeMonte F. Imaging of perineural tumor spread from palatal carcinoma. AJNR Am J Neuroradiol 1998;19:1417-22

5. Ginsberg LE, Eicher SA. Great auricular nerve: anatomy and imaging in a case of perineural tumor spread. AJNR Am J Neuroradiol 2000;21:568-71

6. Lian K, Bartlett E, Yu E. Perineural tumor spread along the sixth cranial nerve: CT and MR imaging. AJNR Am J Neuroradiol 2011;32:E178

7. Majoie CB, Verbeeten B Jr, Dol JA, et al. Trigeminal neuropathy: evaluation with MR imaging. Radiographics 1995;15:795-811

8. Sun X, Liang C, Liu C, et al. Oculomotor paralysis: 3D-CISS MR imaging with MPR in the evaluation of neuralgic manifestation and the adjacent structures. Eur J Radiol 2010;73:221-23

9. Terumitsu M, Seo K, Matsuzawa $\mathrm{H}$, et al. Morphologic evaluation of the inferior alveolar nerve in patients with sensory disorders by high-resolution $3 \mathrm{D}$ volume rendering magnetic resonance neurography on a 3.0-T system. Oral Surg Oral Med Oral Pathol Oral Radiol Endod 2011;111:95-102

10. Chang PC, Fischbein NJ, McCalmont TH, et al. Perineural spread of malignant melanoma of the head and neck: clinical and imaging features. AJNR Am J Neuroradiol 2004;25:5-11

11. Bathla G, Hegde AN. The trigeminal nerve: an illustrated review of its imaging anatomy and pathology. Clin Radiol 2013;68:203-13

12. Borges A, Casselman J. Imaging the trigeminal nerve. Eur J Radiol 2010;74:323-40

13. Cassetta M, Pranno N, Pompa V, et al. High resolution 3-T MR imaging in the evaluation of the trigeminal nerve course. Eur Rev Med Pharmacol Sci 2014;18:257-64

14. Naganawa S, Koshikawa T, Fukatsu H, et al. MR cisternography of the cerebellopontine angle: comparison of three-dimensional fast asymmetrical spin-echo and three-dimensional constructive interference in the steady-state sequences. AJNR Am J Neuroradiol 2001;22:1179-85

15. Sheth S, Branstetter BF, Escott EJ. Appearance of normal cranial nerves on steady-state free precession MR images. Radiographics 2009;29:1045-55

16. Williams LS, Schmalfuss IM, Sistrom CL, et al. MR imaging of the trigeminal ganglion, nerve, and the perineural vascular plexus: normal appearance and variants with correlation to cadaver specimens. AJNR Am J Neuroradiol 2003;24:1317-23

17. Zhang Z, Meng Q, Chen $Y$, et al. 3-T imaging of the cranial nerves using three-dimensional reversed FISP with diffusion-weighted MR sequence. J Magn Reson Imaging 2008;27:454-58

18. Deng W, Chen SL, Zhang ZW, et al. High-resolution magnetic resonance imaging of the inferior alveolar nerve using 3-dimensional magnetization-prepared rapid gradient-echo sequence at 3.0T. J Oral Maxillofac Surg 2008;66:2621-26

19. Mori S, Kaneda T, Fujita Y, et al. Diffusion tensor tractography for the inferior alveolar nerve (V3): initial experiment. Oral Surg Oral Med Oral Pathol Oral Radiol Endod 2008;106:270-74

20. Qin Y, Zhang J, Li P, et al. 3D double-echo steady-state with water excitation MR imaging of the intraparotid facial nerve at 1.5T: a pilot study. AJNR Am J Neuroradiol 2011;32:1167-72

21. Reynolds PA. Face and scalp. In: Standring S, ed. Gray's Anatomy. 40th ed. New York: Elsevier; 2008:541-43

22. Kundel HL, Polansky M. Measurement of observer agreement. Radiology 2003;228:303-08

23. Nemzek WR, Hecht $S$, Gandour-Edwards R, et al. Perineural spread of head and neck tumors: how accurate is MR imaging? AJNR Am J Neuroradiol 1998;19:701-06

24. Schmalfuss IM, Tart RP, Mukherji S, et al. Perineural tumor spread along the auriculotemporal nerve. AJNR Am J Neuroradiol 2002;23: $303-11$ 